\title{
Safety aid elimination as a brief, preventative intervention for social anxiety: A randomized controlled trial in university students
}

Honami Arai ( $\sim$ honamy821@gmail.com )

Chiba University

Shin-ichi Ishikawa

Doshisha University

Sho Okawa

Chiba University

Kohei Kishida

Doshisha University

Kristina Jo Korte

Harvard Medical School - Massachusetts General Hospital

Norman Brad Schmidt

Florida State University

\section{Research Article}

Keywords: Prevention, group intervention, anxiety, social anxiety, safety behavior

Posted Date: November 17th, 2020

DOI: https://doi.org/10.21203/rs.3.rs-107583/v1

License: (c) (i) This work is licensed under a Creative Commons Attribution 4.0 International License. Read Full License 


\section{Abstract}

\section{Background}

Although there are a variety of treatment options for social anxiety, effective prevention strategies for those with subclinical social anxiety are limited. This study evaluated a single session group formatted prevention program focused on the reduction of safety behaviors in both a proof-of-concept study and a randomized controlled trial (RCT).

\section{Methods}

Participants $(N=59)$ were nontreatment seeking Japanese students with high levels of social anxiety. Participants were randomized to either an active treatment focused on identification and elimination of safety behavior or a control group involving the discussion of healthy coping strategies. Both conditions met for 120 minutes in small groups (4 to 6 participants per group). The preliminary proof-of-concept study as well as the RCT demonstrated high levels of acceptability.

\section{Results}

Findings from the RCT indicated significant improvement in social anxiety symptoms compared to HEAL group among those in the active treatment condition at post-treatment, which was maintained at follow-up. In addition, participants in the treatment condition showed significant improvement in depression. The effect size difference in symptoms between conditions was in the large range.

\section{Conclusions}

The present study provides preliminary support for the efficacy of a newly developed treatment program targeting safety behaviors in students with subclinical social anxiety. The present results also illustrate the promise of a brief, indicated prevention strategy focused on safety behavior among those with subclinical social anxiety.

\section{Background}

Social anxiety disorder (SAD) is characterized by excessive fear and avoidance of social and/or performance situations (APA, 2013). The prevalence rate of SAD is approximately $12 \%$, making it one of the most common psychiatric conditions in the world (Kessler et al., 2004). Social anxiety is a frequently reported problem among college students and is associated with other challenges including elevated rates of depression and substance use (Beidel \& Turner, 1998; Panchankis \& Goldfreid, 2006). Furthermore, social anxiety, even at the subsyndromal level, can lead to substantial impairment in social, occupational, and familial domains (Merikangas, Avenevoli, Acharyya, Zhang, \& Angst, 2002; Davidson, Hughes, George, Blazer, 1994; Ruscio, 2010).

Over the last several decades, empirically supported treatments have been developed for anxiety psychopathology including SAD (Chambless \& Ollendick, 2001). Cognitive-behavioral therapy (CBT) is one form of treatment that has been shown to be efficacious for anxiety disorders (Mayo-Wilson et al., 2014). Despite the effectiveness of these treatments, relatively few individuals obtain them due to a number of barriers including logistical and financial constraints (Schleider, Abel, \& Weisz, 2019). Furthermore, treatment response is often incomplete and the prevalence of anxiety disorders including SAD remains high (Kessler et al., 2004; Springer, Levy, \& Tolin, 2018), suggesting a need for increased efforts aimed at their prevention.

Brief interventions for youth mental health problems have shown promise in reducing and preventing anxiety (Cardamone-Breen et al., 2018). Given that a majority of adolescents with significant anxiety problems go without services (Kataoka, Zhang, \& Wells, 2002), brief interventions may serve as helpful alternatives or adjuncts to traditional, 
multi-session psychotherapy. Korte \& Schmidt (2020) developed a single-session, intervention specifically focused on mitigating subclinical social anxiety in college students. This intervention was based on the elimination of safety behavior, that is, strategies used to manage or cope with anxiety. The intervention, "Safety Aid Elimination Intervention" (SAFE), is a one-session, 120 min program targeting safety behaviors. This brief preventative intervention was based on treatment studies that utilize a similar approach of focusing on the identification and elimination of safety behavior among those with clinically significant anxiety (Riccardi, Korte, \& Schmidt, 2017; Schmidt et al., 2012).

The utilization of safety behavior is believed to play a key role in accelerating the development and maintenance of anxiety (Salkovskis, 1991). In the context of perceived threat, some individuals may rely on so-called safety behavior to help them manage their anxiety. These strategies often help reduce anxiety in the short term, but they can contribute to increased anxiety by essentially validating erroneous threats (Helbig-Lang \& Peterman, 2010). Moreover, as the developmental trajectory progresses, an individual may increasingly utilize safety behavior, which further strengthens these fears. Prior work indicates that False Safety Behavior Elimination Therapy (F-SET) is effective when delivered in a group as well as an individual format to patients with anxiety psychopathology (Riccardi et al., 2017; Schmidt et al., 2012).

This prior work led to an adaptation of the protocol to evaluate whether it might also be effective as a preventative intervention for those experiencing subclinical anxiety (Korte et al., 2020). The rationale for this adaptation is consistent with theory suggesting that the use of safety behavior may play a role in the development of anxiety disorders in those experiencing subclinical levels of anxiety (Deacon \& Maack, 2008). Korte et al. (2020) tested the efficacy of SAFE in a randomized controlled trial (RCT) with university students who had elevated anxiety symptoms. Participants were randomized to either a one session of SAFE or a health-focused control group. Results revealed significant between group differences in the reduction of social anxiety, worry, and levels of impairment in the active intervention group relative to the control group. Furthermore, change in safety aid utilization was a significant mediator of outcomes. Despite the promising findings in this initial trial, SAFE was delivered in an individual format, thereby limiting the potential cost effectiveness of a group delivered preventative intervention.

The current study is the first study to extend SAFE in the individual format to group format. There is an increasing demand for mental health services and professionals in Japan, especially in educational institutions, and a group intervention offers a more efficient and cost-effective way to provide quality mental health care to a larger number of students (Chiumento et al., 2017; Rith-Najarian et al., 2019). Moreover, one strength of group formats for intervening with social anxiety is that a group setting necessarily promotes social exposure and interaction (Taube-Schiff, Suvak, Antony, Bieling, \& McCabe., 2007). We were interested in determining whether a group-based, single session of SAFE could be effective in reducing student's social anxiety as well as related forms of psychopathology (i.e., depression).

Based on prior work, we conducted a proof of concept (pilot) open trial followed by a randomized controlled trial of SAFE. The proof of concept study was designed to evaluate the acceptability of the adapted SAFE protocol in a small cohort of college students with elevated social concerns. It was hypothesized that the protocol would be acceptable (based on low dropouts) and efficacious based on reduction in targeted outcomes (e.g., social anxiety symptoms). This study was followed with a 2 (SAFE vs. heathy coping control group) $\times 3$ (pre, post treatment, and one-month follow up) randomized study design in college students with elevated social anxiety. We hypothesized that SAFE would lead to greater reduction in the primary symptom outcome (social anxiety symptoms) as well as secondary outcomes (depression and social impairment) at a one week and one-month follow-up compared to control group. Consistent with Korte and Schmidt (2020) we also hypothesized that the changes in safety behavior would mediate the association between the experimental conditions and social anxiety symptoms at follow up.

\section{Methods}




\section{Proof of Concept Study}

Before the RCT, we first conducted a pilot study with six students to determine acceptability and provide initial efficacy data. Acceptability was assessed based on dropout rates as well as using a brief questionnaire rated on a 5-point Likert Scale (Shiraishi \& Koshikawa, 2010), assessing participants' subjective perceptions of the program (e.g., understandability, user-friendliness). In addition, a short battery of self-report measures was completed before and after the program as well as at a one-month follow up period to assess symptom change. The primary outcome measure was the Japanese version of the Social Interaction Anxiety Scale (SIAS) since it has demonstrated stronger correlations than the SPS with other social anxiety scales (Brown et al., 1997; Heimberg et al., 1992). Secondary outcomes were scores on the Japanese version of Social Phobia Scale (SPS; Kanai et al., 2004), the Japanese version of Beck Depression Inventory (BDI-Il; Kojima, Furukawa, Takahashi, Kawai, Nagaya \& Tokudome., 2003), the Japanese version of Sheehan Disability Scale (SDS; Yoshida et al., 2004), and the Japanese version of Subtle Avoidance Frequency Examination (SB; Arai et al., 2015). Study participants were recruited from university classes and received $\backslash 6000$ in compensation equivalent to $\$ 50.00$. A total of six people took the group program, Fifty percent of participants were women, and had a mean age of 25.17 (SD = 5.04). Consistent with high levels of acceptability, there were no dropouts during the intervention or follow up. In addition, SAFE was rated positively on the acceptability questionnaire (How well did you understand the program materials? : $M=4.67$, Was it worth your time participating in the program?: $M=3.83$, Do you think the content of the program is easy to include in your daily life?: $M=3.50$, How much did the program burden to you: $M=3.50$, Do you want to continue what you learned in the program?: $M=3.83$ ). Preliminary efficacy data were also consistent with expectation (see Table 1) in indicating reductions in the primary and secondary outcomes.

\section{Randomized Controlled Trial}

\section{Participants}

We recruited participants through the psychology department's undergraduate research pool. Inclusion criteria were (1) students 18 years older, (2) total scores in the Social Interaction Anxiety Scale (SIAS; Kanai et al., 2004) of 40 or higher. Exclusion criteria were (1) reporting suicidal ideation, (2) receiving current pharmacological or psychological treatments. Individuals who signed up for the research study received $\backslash 6000(\$ 50.00)$ in compensation.

\section{Study Design}

This randomized controlled trial used a parallel-group design. An independent researcher generated random allocation sequences using a computer and stratified randomization by sex. Allocation ratio was 1:1 to SAFE or active control. All procedures performed in studies involving human participants were in accordance with the ethical standards and approved by the institutional review board of the first author (\#17063). The trial was pre-registered in the UMIN clinical trials (UMIN000032064). The recruitment of the trial was started on 6th April 2018 and the follow-up was completed on 7th July 2018 based on completion of prerequisite sample size. Based on a power analysis using the mean reduction of SIAS from pre to one-month follow up reported by Korte et al. (2020), using G*Power (Faul, Erdfelder, Buchner, \& Lang, 2009), the required sample size was calculated as $N=28$ for a single arm when power $1-\beta$ was 0.90 and type 1 error was $5 \%$. Therefore, we were determined to recruit 30 participants per cell to be adequately powered to evaluate the primary study outcome variable.

\section{Assessment Measures}




\section{Primary outcome}

The primary outcome was social anxiety symptoms, measured using the total scores of the Japanese version of SIAS which contains 20 items. The SIAS assesses general fears of social interaction. The SIAS has been found to be a reliable measure of social anxiety, exhibiting good convergent and discriminate validity (Brown et al, 1997; Heimberg et al. 1992). The SIAS demonstrated good reliability at baseline $(a=.78)$, and Month $1(a=.79)$. Internal consistency of the SIAS was .86 in the current sample.

\section{Secondary outcomes}

The secondary outcomes were scores on the SPS (Kanai et al., 2004), the Japanese version of BDI-II (Kojima et al., 2003), the Japanese version of SDS (Yoshida et al., 2004), and the Japanese version of SB (Arai et al., 2015). The Internal consistency of the SPS, the BDI-II, the SDS, and the SB in the current sample were $.82, .83, .79, .86$ respectively.

\section{Acceptability of the intervention}

Acceptability was based on relative dropout rates as well as homework compliance rates. Dropout rates of CBT in controlled trials for anxiety disorders are typically 5.6-19.0\% (Otto, Smits, \& Reese, 2004). Therefore, we set an acceptable dropout rate threshold of lower than $12 \%$. For safety, we set a threshold of zero severe adverse events for SAFE.

\section{Procedure}

Screening Appointment. Upon arrival, participants first read and signed an informed consent form. Then they were asked to complete a self-report battery of questionnaires. They then completed the MINI and additional self-report questionnaires to determine their eligibility. Participants that did not meet the inclusionary criteria, were debriefed, thanked for their time and dismissed from the study. Those who met inclusionary criteria were asked their contact address and the study coordinator contacted them later to adjust the schedule to join the intervention. Eligible participants were randomly assigned to one of the two intervention conditions either the Safety Aid Fading and Eliminating Intervention (SAFE) condition or the Health Education and Adaptive Living (HEAL) condition. Participants were blinded which allocation they were assigned.

Intervention Appointment. Participants were provided with materials from the intervention, homework sheets, and extra reading materials. To control for level of participation across experimental conditions, the number of group discussions and the number of writing worksheets were matched in both groups. Both interventions were delivered by the first author, who is a clinical psychologist with training in CBT for anxiety disorders. The first author was trained on the SAFE protocol by the authors of the original SAFE protocol (Korte et al., 2020). Following completion of the intervention, participants were scheduled for their one-week check-in appointment.

One-Week Check-In Appointment. The researcher sent an e-mail to the participants one day before their one-week check-in appointment, to remind them of the appointment time and to bring completed homework forms. Upon arrival, participants were asked to complete the one-week self-report questionnaire battery. The homework forms were collected at the checkin, and participants also had the opportunity to privately discuss with the therapist any questions they had about their homework for the coming week. If a participant needed guidance on their homework, the therapist provided assistance. Time spent with a therapist was kept to a minimum with the typical amount of interaction being under 5 minutes. The therapist encouraged the participants to continue their homework exercises after one -week post toward one-month FU.

One-Month Follow-Up. One-month follow-up questionnaires were collected online.

Page 5/19 


\section{Description of experimental conditions. SAFE active group}

SAFE is modeled on the F-SET manual (Schmidt et al. 2012). In F-SET, safety behaviors are conceptualized as behavioral and cognitive coping strategies that an individual engages in to manage anxiety. Although these behaviors may help to relieve anxiety in the short-term, they often exacerbate anxiety in the long-term. F-SET, therefore, outlines an educational and behavioral protocol commonly employed in the treatment of anxiety disorders. The SAFE intervention was designed as a two-hour session to ensure that it would be relatively brief, while also providing enough time to cover the key topics in the F-SET manual. The intervention session is comprised of two parts. The first part focuses on providing psychoeducation on anxiety. A conceptual model of anxiety, which includes all anxiety disorders, is explained. Fear is explained as a memory structure (i.e., a fear structure) that is based on personal history. Participants are also informed of the emotional processing model of anxiety, and they learn to identify their false alarms resulting from various stimuli. The session then transitions to the second part that focuses on safety behavior. Consistent with the F-SET protocol, the participants learn to identify and fade their false safety behavior. For example, a participant reporting avoidance of asking their teachers questions because of a fear of negative evaluation, might focus on this behavior by first asking a question after class. An individualized plan would then be created that included first asking questions outside of class or on the phone or email and then progressing to asking in class.

Another key component of F-SET is the utilization of antiphobic exercises. Antiphobic exercises are behaviors in direct opposition to the participant's phobic tendencies (i.e., doing the opposite of what they fear). Whereas having a phobic attitude involves avoiding stimuli that may trigger a false fear structure, having an antiphobic attitude involves adopting attitudes and behaviors that are designed to purposefully bring about the feared situation. For example, a person who is afraid of saying something wrong would be instructed to intentionally misspeak or give incorrect answers. To develop an antiphobic attitude, the therapist assisted participants in incorporating antiphobic behaviors as part of their weekly homework. The participants were provided with self-monitoring forms to track their progress over the follow up check-in appointments.

\section{Manual translation process of the programs}

We developed a Japanese version of the SAFE manual that was semantically equivalent to the original by following a translation/back-translation procedure. First, the first author translated the original version into Japanese. Second, Japanese psychologists modified some of the words and phrases in the draft of the Japanese version. Third, two professional translators and a professional proofreader, who did not participate in the Japanese translation, backtranslated the modified Japanese version into English. Fourth, the authors of the original manual compared the original and back-translated versions and confirmed that the two versions were essentially equivalent. To carry out SAFE in a group format, the contents of the program manual were converted to PowerPoint slides. We develop the HEAL control program using the same process.

\section{HEAL control group}

To control for time and attention with the experimenter across experimental groups, control participants (HEAL) attended a health-focused group lecture. The HEAL manual was developed based on the control program utilized in the previous study (Korte et al., 2020). Psychoeducation was provided by the same therapist conducting the SAFE group. The control condition took place over one session and lasted for approximately two hours. The intervention session was separated into two parts. The first part focused on the importance and benefits of good nutrition. Participants were asked to recall what they had eaten for breakfast, lunch, and dinner, and they scored the meals according to a nutrition table. The therapist then provided guidelines for achieving healthy diet habits. The second part covered sleep habits, and the 
therapist discussed how students could monitor and improve sleep habits themselves. After the session, participants were given nutrition and sleep-monitoring forms and instructed to fill in the forms daily for the next week.

\section{Data Analysis}

All analyses were done on the intent-to-treat (ITT) sample. The SAFE and HEAL groups were compared at baseline on demographic and clinical outcomes using between-subjects analyses of variance (ANOVAs) and chi square analysis.

The first hypothesis was that the SAFE intervention would lead to greater reductions in social anxiety symptoms, depression and social impairment at a one week and one-month follow-up compared to HEAL intervention. To examine the first hypothesis, we conducted a linear mixed model with a maximum likelihood estimator and calculated an effect size. We first compared a random intercept model and random intercept and slope model, and the best fit was the random intercept and slope model. Therefore, we conducted the linear mixed analysis using a model with a random intercept and slope. We set the group (SAFE, HEAL), time (pre, post, follow-up), and interaction (group by time) as fixed effects. Participants were entered as random effects. The effect size of Hedge's g and 95\% confidence intervals (Cl) were calculated for pre to post and pre to follow-up changes on clinical outcomes. Missing data were handled by the lastobservation-carried-forward method. The linear mixed model, the effect size of Hedge's, and $95 \% \mathrm{Cl}$ were calculated using STATA 16. The mediation analyses were conducted using Mplus 8.4.

We conducted the mediation analyses using maximum likelihood estimation to examine the role of safety behavior change as mediating differences between social anxiety change for the SAFE and HEAL groups. We estimated biascorrected bootstrapped confidence intervals (Cls) using 5,000 bootstrap samples for indirect effects. The effect was interpreted as significant if the $95 \% \mathrm{Cls}$ did not include zero.

\section{Results}

\section{Baseline characteristics}

A total of 127 individuals were recruited to participate in this study. Of the 127 participants, 68 did not enroll in the study As such, fifty-nine participants were randomized to either the SAFE active group $(n=26)$ or the HEAL control group ( $n=$ 33). A majority of the participants were women with a mean age of $19.82(S D=.81) .10 \%$ of the participants met criteria for SAD according to the M.I.N.I. No participant has reported current or in the past of other mental health disorders. There were no significant differences between two conditions in terms of age, $F(1,57)=2.74, p=.1$ or gender, $\chi^{2}(1)=0.03, p=.87$. In addition, all the baseline clinical characteristics were compared between groups and there were no significant differences in percent meeting for diagnostic status or symptoms: SIAS: $F(1,57)=0.73$, SPS: $F(1,57)=.01, \mathrm{BDI} F(1,57)=0.21$, SAFE: $F(1,57)=0.19$. The CONSORT diagram of enrollment is shown in Figure 1 .

Homework compliance, Dropout rates and adverse events

Homework completion was generally good with $85 \%$ of SAFE group participants completing home practice assignments at week 1. In HEAL, $100 \%$ of participants completed home practice assignments at week 1 . There were no dropouts in either conditions as well as no adverse events.

\section{Primary and secondary outcomes}

The descriptive statistics for primary and secondary outcome measures are shown in Table 2. The interaction (group by time) for each outcome is shown in Table 3. In the primary analyses to examine the SIAS change score, a significant 
interaction between group and time was found (Figure 2). The estimates showed the decrease in SIAS score from pre to one-week post in the SAFE group was significantly larger than in the HEAL group. Similarly, the decrease in SIAS score from pre to one-month follow-up in the SAFE group was significantly greater than in the HEAL group suggesting durability of the intervention effects. For the secondary outcome measures, findings for each outcome measure are reported in Table 3. There were no significant interactions between group and time in any of the secondary outcome measures. The decrease in BDI-II score from pre to follow-up for SAFE group was greater than in the HEAL group, but it did not reach statistical significance $(p=.07)$. The pre to follow-up change in SB scores between the SAFE and HEAL groups was also trending but not significant $(p=.06)$.

\section{Effect sizes}

Table 4 shows effect sizes (Hedge's g) between pre to one-week post and pre to one month follow-up for each group. For pre to one-week post, the effect size for SIAS and BDI-II were moderate to large in SAFE group while the effect size for all the outcomes were small in HEAL group. For pre to one-month follow-up, the effect sizes for the SIAS, SPS, BDI, and SB in the SAFE group were moderate to large. For the HEAL group, only SPS had a large effect size and effect size of all the other outcomes were small.

\section{Mediation Analyses}

We also hypothesized that change in safety behavior would mediate the association between intervention conditions (SAFE vs. HEAL) and reduction in social anxiety symptoms, as measured by the SIAS. To establish temporal precedence of the mediator, we examined whether change in safety behavior from pre- to post-treatment mediated the relationship between condition and social anxiety symptom change from pre- to follow-up assessment. Following the procedure outlined by Preacher and Hayes (2008) we tested the products of (1) the independent variable (Group: SAFE, HEAL) to the mediator (SB change pre- to post-treatment) ( $a$ path: unstandardized beta $=3.11, \mathrm{SE}=3.10,95 \% \mathrm{Cl}:-1.82 \sim 8.33)$, and (2) the mediator to the dependent variable (change in SIAS scores pre- to follow-up) when the independent variable is taken into account ( $\beta$ path: unstandardized beta $=0.65, \mathrm{SE}=0.11,95 \% \mathrm{Cl}=0.46 \sim 0.81$ ). Results revealed that the $95 \%$ confidence interval of the indirect path $(\mathrm{a} \beta)$ overlapped with zero for reduction in SIAS (lower limit $=-1.05$, upper limit $=$ 5.37), indicating no mediational effect.

We also computed a test of simple mediation to examine (1) the independent variable (Group: SAFE, HEAL) to the mediator (SB change pre- to one-month FU) ( $a$ path: unstandardized beta $=-6.37, \mathrm{SE}=3.27,95 \% \mathrm{Cl}$ : 0.95 11.87)), and (2) the mediator to the dependent variable (change in SIAS scores pre- to follow-up) when the independent variable is taken into account ( $\beta$ path: unstandardized beta $=0.51, \mathrm{SE}=0.09,95 \% \mathrm{Cl}: 0.38 \sim 0.66$ ). Results revealed that the $95 \%$ confidence interval of the indirect path $(a \beta)$ did not overlapped with zero for reduction in SIAS (lower limit $=0.50$, upper limit $=6.48$ ), indicating a mediation effect. Thus, change in SB scores from pre- to one month-treatment mediated the relationship between conditions and reduction in social anxiety symptoms.

\section{Discussion}

This RCT study was designed to examine the feasibility and efficacy of a brief group prevention program for subclinical social anxiety. Despite the brevity of the intervention, significant reductions in social anxiety symptoms were evident one week following the group session and these reductions appear to be durable up to one month following the intervention. These favorable outcomes in terms of the primary outcome were comparable to the previous study (Korte \& Schmidt, 
2020) showing significant reductions in SIAS. However, Korte and Schmidt did not find one-week group differences, as these were only apparent at one month. One explanation for these differences is that the group format may foster more rapid change in social anxiety than would the individual format. Our group model also incorporated required discussions in a social setting, as well as the implicit social exposure involved in the two-hour group meeting. Previous work has suggested that group interventions for social anxiety helped reduce a sense of isolation and fostered solidarity (TaubeSchiff et al., 2017). The group may help normalize experiences and provide a ready-made social format in which to take risks and break maladaptive habits (Fogarty, Hevey, \& McCarthy, 2019).

Findings were less consistent with regard to the secondary outcomes. More specifically, there were no statistically significant group differences despite evidence for substantial effect sizes in the SAFE group. For example, within group effect sizes for pre to one-month follow-up was large in SPS, BDI-II, and SB in SAFE group. This level of improvement for the SAFE intervention is comparable to effects seen in more elaborate CBT protocols for social anxiety (Goldin, Morrison, Jazaieri, Brozovich, Heimberg, \& Gross, 2016). Failure to find condition differences on these measures is partly due to a lack of power as well as partly due to the control condition showing substantial reductions in some measures, particularly the SPS and the BDI-II. Such effects for the control condition may simply be due to expectancy or social desirability (Boot, Simons, Stothart, and Stutts, 2013). It is also possible, however, that the active control produced real effects either from the social exposure that was inherent to attending a group or to the content of the HEAL protocol, which includes a variety of healthy lifestyle techniques such as increasing exercise or sleep hygiene, which have been shown to positively affect mood and anxiety (Petruzzello et al., 1991; Conn et al., 2011; Craft \& Landers, 1998).

The mediation hypothesis was partially supported. Unfortunately, temporal mediation was not supported whereas change in safety behaviors at one month was consistent with mediation. Of note, this pattern of effects is consistent with the relative level of change in safety behaviors noted in this group, which generally suggested that these behaviors did not change very rapidly. There was very little change at one week and more substantial movement at one month. This rate of change is consistent with previous work on safety behaviors. For example, Korte, Norr, \& Schmidt (2018) suggested that it is common for individuals to become more aware of their safety behavior during the process of learning about and eliminating them. These authors suggest that it is not unusual for patients to discover new safety behavior at the beginning stages of treatment. In hindsight, it would have been better to assess safety behaviors after two to three weeks to provide more opportunity for safety aid reduction to take place and still allow for a test of temporal mediation. Thus, safety behavior score at one month FU was significantly lower than one-week treatment.

Findings were also consistent with an intervention showing good acceptability based on self-report, homework compliance and dropout. The negligible dropout rate in this trial was consistent with other SAD prevention efforts (9\%) (Bijorson et al., 2011) as well as traditional CBT (5.6\%-19.0\%) (Otto et al., 2004). This result is also consistent with a previous study (Korte et al., 2020), in which dropout rate was $4 \%$. These results speak to the viability of SAFE in university settings.

As with any study, there are a number of limitations to consider. First, participants in these studies were highly educated university students. This was by no means a diverse sample, and it is unclear whether the SAFE program would work effectively for youth with less education. Future studies should recruit subclinical participants in other settings, such as community settings, is an important next step. Second, this study only relied on self-report data as primary and secondary outcomes; it would have been preferable to have behavioral outcomes such as a public speaking task (Allen, Kennedy, Dockray, Cryan, Dinan, \& Clarke, 2016) to get a broader picture of the treatment effects. Third, this study did not include a long-term follow up. Thus, it is unclear whether symptom reduction would be maintained for a longer duration. Relevant to the brief intervention design, occasional long-term prevention sessions, so-called booster sessions, might be incorporated to further enhance efficacy or reduce the likelihood of relapse of anxiety symptoms (Christensen, Pallister, Smale, Hickie, \& Calear, 2010; Gearing et al., 2013; Neil \& Christensen, 2009). 
Because a mental health professional led the SAFE group, it is unclear whether the efficacy of SAFE would differ if lay professionals or educators led the group. Because SAFE is designed as a selected preventative intervention (Feldner, Zvolensky, \& Schmidt, 2004) that could be widely implemented, using lay professionals is one method of making such interventions more scalable. Such approaches seem promising as educators have provided school-based CBT in with promising results (Stallard et al., 2014; Teubert \& Pinquart, 2011). One advantage of SAFE relative to other CBT approaches is that it is relatively simple and straightforward. In the future work, it would be interesting to evaluate whether non-clinicians can effectively deliver SAFE with minimal training.

In summary, the findings of the current study support the use of a single session group intervention designed to treat subclinical social anxiety. Despite the brevity of the intervention, SAFE shows promise in improving social anxiety symptoms. However, further research is warranted to determine the robustness of the current findings, and explore ways to disseminate this promising intervention.

\section{Declarations}

Conflict of Interest: The authors declare no conflict of interest

\section{References}

Allen, A. P., Kennedy, P. J., Dockray, S., Cryan, J. F., Dinan, T. G., \& Clarke, G. (2016). The Trier

Social Stress Test: Principles and practice. Neurobiology of stress, 6, 113-126.

https://doi.org/10.1016/j.ynstr.2016.11.001

American Psychiatric Association. (2013). Diagnostic and statistical manual of mental disorders

(5th ed.). Washington, D.C: American Psychiatric Press.

Arai, H., Aoki, S., Kanai, Y., \& Sakano, Y. Psychometric properties of the Japanese version of the subtle avoidance frequency examination (SAFE-J): Factor structure, reliability, and validity in Japanese college students. Japanese Journal of Archives of psychiatric diagnostics and clinical evaluation, 8, 114-125.

Beidel, D. C., \& Turner, S. M. (1998). Shy children, phobic adults: Nature and treatment of social phobia. American Psychological Association. https://doi.org/10.1037/10285-000

Bjornsson, A. S., Bidwell, L. C., Brosse, A. L., Carey, G., Hauser, M., Mackiewicz Seghete, K. L., Schulz-Heik, R. J., Weatherley, D., Erwin, B. A., \& Craighead, W. E. (2011). Cognitive behavioral group therapy versus group psychotherapy for social anxiety disorder among college students: a randomized controlled trial. Depression and anxiety, 28(11), 1034-1042. https://doi.org/10.1002/da.20877

Boot, W. R., Simons, D. J., Stothart, C., \& Stutts, C. (2013). The Pervasive Problem With Placebos in Psychology: Why Active Control Groups Are Not Sufficient to Rule Out Placebo Effects. Perspectives on psychological science: a journal of the Association for Psychological 
Science, 8(4), 445-454. https://doi.org/10.1177/1745691613491271

Brown, E. J., Turovsky, J., Heimberg, R. G., Juster, H. R., Brown, T. A, \& Barlow, D. H. (1997).

Validation of the Social Interaction Anxiety Scale and the Social Phobia Scale across the anxiety disorders. Psychological Assessment, 9(1), 21-27 https://doi.org/10.1037/10403590.9.1.21

Brown, T. A., Campbell, L. A., Lehman, C. L., Grisham, J. R., \& Mancill, R. B. (2001). Current and lifetime comorbidity of the DSM-IV anxiety and mood disorders in a large clinical sample. Journal of Abnormal Psychology, 110(4), 585-599. https://doi:10.1037//0021-843x.110.4.585

Cardamone-Breen, M. C., Jorm, A. F., Lawrence, K. A., Rapee, R. M., Mackinnon, A. J., \& Yap, M. (2018). A Single-Session, Web-Based Parenting Intervention to Prevent Adolescent Depression and Anxiety Disorders: Randomized Controlled Trial. Journal of medical Internet research, 20(4), e148. https://doi.org/10.2196/jmir.9499

Conn, V. S., Hafdahl, A. R., \& Mehr, D. R. (2011). Interventions to increase physical activity among healthy adults: meta-analysis of outcomes. American journal of public health, 101(4), 751-758. https://doi.org/10.2105/AJPH.2010.194381

Craft, L. L., \& Landers, D. M. (1998). The effect of exercise on clinical depression and depression resulting from mental illness: A meta-analysis. Journal of Sport \& Exercise Psychology, 20(4), 339-357. https://doi.org/10.1123/jsep.20.4.339

Chambless, D. L., \& Ollendick, T. H. (2001). Empirically supported psychological, interventions: Controversies and evidence. Annual Review of Psychology, 52, 685-716. https://doi:DOI 10.1146/annurev.psych.52.1.685

Chiumento, A., Hamdani, S. U., Khan, M. N., Dawson, K., Bryant, R. A., Sijbrandij, M., Nazir, H., Akhtar, P., Masood, A., Wang, D., van Ommeren, M., \& Rahman, A. (2017). Evaluating effectiveness and cost-effectiveness of a group psychological intervention using cognitive behavioural strategies for women with common mental disorders in conflict-affected rural Pakistan: study protocol for a randomised controlled trial. Trials, 18(1), 190. https://doi.org/10.1186/s13063-017-1905-8

Christensen, H., Pallister, E., Smale, S., Hickie, I. B., \& Calear, A. L. (2010). Community-based prevention programs for anxiety and depression in youth: a systematic review. Journal of 
Primary Prevent, 31, 139-170. https://doi.org/10.1007/s10935-010-0214-8

Davidson, J. R., Hughes, D. C., George, L. K., \& Blazer, D. G. (1994). The boundary of social phobia. Exploring the threshold. Archives of general psychiatry, 51(12), 975-983.

https://doi.org/10.1001/archpsyc.1994.03950120047008

Deacon, B., \& Maack, D. J. (2008). The effects of safety behaviors on the fear of contamination: an experimental investigation. Behavior Research and Therapy, 46(4), 537-547.

https://doi:10.1016/j.brat.2008.01.010

Faul, F., Erdfelder, E., Buchner, A., \& Lang, A. G. (2009). Statistical power analyses using G*Power

3.1: tests for correlation and regression analyses. Behavior research methods, 41(4), 11491160 https://doi.org/10.3758/BRM.41.4.1149

Feldner, M.T., Zvolensky, M.J., \& Schmidt, N.B. (2004). Prevention of anxiety psychopathology: A critical review of the empirical literature. Clinical Psychology: Science and Practice, 11, 405-424 .https://doi.org/10.1093/clipsy.bph098

Fogarty, C., Hevey, D., \& McCarthy, O. (2019). Effectiveness of cognitive behavioural group therapy for social anxiety disorder: long-term benefits and aftercare. Behavioural and cognitive psychotherapy, 47(5), 501-513.

Goldin, P. R., Morrison, A., Jazaieri, H., Brozovich, F., Heimberg, R., \& Gross, J. J. (2016). Group CBT versus MBSR for social anxiety disorder: A randomized controlled trial. Journal of consulting and clinical psychology, 84(5), 427-437. https://doi.org/10.1037/ccp0000092 Heimberg, R. G., Mueller, G. P., Holt, C. S., Hope, D. A., \& Liebowitz, M. R. (1992). Assessment of anxiety in social interaction and being observed by others: The Social Interaction Anxiety Scale and the Social Phobia Scale. Behavior Therapy, 23(1), 53-73 https://doi.org/10.1016/S0005-7894(05)80308-9

Heimberg, R. G., Stein, M. B., Hiripi, E., \& Kessler, R. C. (2000). Trends in the prevalence of social phobia in the United States: A synthetic cohort analysis of changes over four decades. European Psychiatry, 15(1), 29-37. https://doi.org.10.1016/S0924-9338(00)00213-3 Helbig-Lang, S., \& Petermann, F. (2010). Tolerate or eliminate? A systematic review on the effects of safety behavior across anxiety disorders. Clinical Psychology: Science and Practice, 17, 218-233.

Kataoka, S. H., Zhang, L., \& Wells, K. B. (2002). Unmet need for mental health care among U.S. 
children: variation by ethnicity and insurance status. The American journal of

psychiatry, 159(9), 1548-1555. https://doi.org/10.1176/appi.ajp.159.9.1548

Kanai, Y., Sasagawa, S., Junwen, C., Suzuki, S., Shimada, H., \& Sakano, Y. (2004). Development and Validation of the Japanese Version of Social Phobia Scale and Social Interaction Anxiety Scale, Japanese Journal of Psychosomatic Medicine, 44(11), 841-850.

Kawakami, N., Takeshima, T., Ono, Y., Uda, H., Hata, Y., Nakane, Y., ... Kikkawa, T. (2005).

Twelve-month prevalence, severity, and treatment of common mental disorders in communities in japan: Preliminary finding from the world mental health japan survey 2002-2003. Psychiatry and Clinical Neurosciences, 59(4), 441-452. https://doi:10.1111/j.1440-1819.2005.01397.x

Kessler, R. C., Petukhova, M., Sampson, N. A., Zaslavsky, A. M., \& Wittchen, H.-U. (2012).

Twelve-month and lifetime prevalence and lifetime morbid risk of anxiety and mood disorders in the United States. International Journal of Methods in Psychiatric Research, 21(3), 169-184. https://doi.org.10.1002/mpr.1359

Kojima, M., Furukawa, T. A., Takahashi, H., Kawai, M., Nagaya, T., \& Tokudome, S. (2002). Crosscultural validation of the Beck Depression Inventory-II in Japan. Psychiatric Research, $110,291-299$.

Korte, K. J., Norr, A. M., \& Schmidt, N. B. (2018). Targeting Safety Behaviors in the Treatment of Anxiety Disorders: A Case Study of False Safety Behavior Elimination Treatment. American journal of psychotherapy, 71(1), 9-20. https://doi.org/10.1176/appi.psychotherapy.20180001

Korte, J. K., \& Schmidt, N. B. (2020). Transdiagnostic preventative intervention for subclinical anxiety: Development and initial validation. Journal of Psychiatric Research, 126, 34-42. doi.org/10.1016/j.jpsychires.2020.04.001

Mayo-Wilson, E., Dias, S., Mavranezouli, I., Kew, K., Clark, D. M., Ades, A. E., \& Pilling, S. (2014). Psychological and pharmacological interventions for social anxiety disorder in adults: a systematic review and network meta-analysis. Lancet Psychiatry, 1(5), 368-376. https://doi:10.1016/S2215-0366(14)70329-3

Merikangas, K. R., Avenevoli, S., Acharyya, S., Zhang, H., \& Angst, J. (2002). The spectrum of social phobia in the Zurich cohort study of young adults. Biological Psychiatry, 51(1), 81-91. 
https://doi.org.10.1016/S0006-3223(01)01309-9

Neil, A. L., \& Christensen, H. (2009). Efficacy and effectiveness of school-based prevention and early intervention programs for anxiety. Clinical psychology review, 29(3), 208-215. https://doi.org/10.1016/j.cpr.2009.01.002

Otto, M. W., Smits, J. A., \& Reese, H. E. (2004). Cognitive-behavioral therapy for the treatment of anxiety disorders. The Journal of clinical psychiatry, 65 Supp/ 5, 34-41.

Pachankis, J. E., \& Goldfried, M. R. (2006). Social anxiety in young gay men. Journal of Anxiety Disorders, 20(8), 996-1015. https://doi.org/10.1016/j.janxdis.2006.01.001

Petruzzello, S. J., Landers, D. M., Hatfield, B. D., Kubitz, K. A., \& Salazar, W. (1991). A metaanalysis on the anxiety-reducing effects of acute and chronic exercise. Outcomes and mechanisms. Sports medicine (Auckland, N.Z.), 11(3), 143-182. https://doi.org/10.2165/00007256-199111030-00002

Preacher, K. J., \& Hayes, A. F. (2008). Asymptotic and resampling strategies for assessing and comparing indirect effects in multiple mediator models. Behavior Research Methods, 40(3), 879-891. https://doi.org/10.3758/BRM.40.3.879

Riccardi, C. J., Korte, K. J., \& Schmidt, N. B. (2017). False safety behavior elimination therapy: A randomized study of a brief individual trans diagnostic treatment for anxiety disorders. Journal of Anxiety Disorders, 46, 35-45. https://doi.org/10.1016/j.janxdis.2016.06.003

Rith-Najarian, L. R., Mesri, B., Park, A. L., Sun, M., Chavira, D. A., \& Chorpita, B. F. (2019). Durability of Cognitive Behavioral Therapy Effects for Youth and Adolescents With Anxiety, Depression, or Traumatic Stress:A Meta-Analysis on Long-Term Follow-Ups. Behavior Therapy, 50(1), 225-240. https://doi:10.1016/j.beth.2018.05.006

Ruscio A. M. (2010). The latent structure of social anxiety disorder: consequences of shifting to a dimensional diagnosis. Journal of abnormal psychology, 119(4), 662-671. https://doi.org/10.1037/a0019341

Salkovskis, P. M. (1991). The importance of behaviour in the maintenance of anxiety and panic: A cognitive account. Behavioural Psychotherapy, 19, 6-19.

https://doi.org/10.1017/S0141347300011472

Page 14/19 
Schleider, J. L., Abel, M. R., \& Weisz, J. R. (2019). Do Immediate Gains Predict Long-Term

Symptom Change? Findings from a Randomized Trial of a Single-Session Intervention for

Youth Anxiety and Depression. Child psychiatry and human development, 50(5), 868-881.

https://doi.org/10.1007/s10578-019-00889-2

Schmidt, N. B., Bucker, J. D., Woolaway-Bickel, K., Preston, J. L., \& Norr, A. (2012). Randomized

Controlled Trial of False Safety Behavior Elimination Therapy: A Unified Cognitive Behavioral

Treatment for Anxiety Psychopathology. Behavior Therapy, 43, 518-532. https://doi:

10.1016/j.beth.2012.02.004.

Shiraishi, S., \& Koshikawa, F. (2010). The effects and issues of mental health interventions for undergraduates. Bulletin of the Integrated Research Center for Educational Practice, Utsunomiya University, 33, 153-160.

Springer, K. S., Levy, H. C., \& Tolin, D. F. (2018). Remission in CBT for adult anxiety disorders: A meta-analysis. Clinical psychology review, 61, 1-8. https://doi.org/10.1016/j.cpr.2018.03.002

Stallard, P., Skryabina, E., Taylor, G., Phillips, R., Daniels, H., Anderson, R., \& Simpson, N. (2014).

Classroom-based cognitive behaviour therapy (FRIENDS): a cluster randomised controlled trial to Prevent Anxiety in Children through Education in Schools (PACES). Lancet Psychiatry, 1(3), 185-192. https://doi:10.1016/S2215-0366(14)70244-5

Taube-Schiff, M., Suvak, M, K., Antony, M.M., Bieling, P.J., \& McCabe, R. E. (2007). Group cohesion in cognitive-behavioral group therapy for social phobia. Behavior Research and Therapy, 45(4), 687-698. https://doi:10.1016/j.brat.2006.06.004

Teubert D, Pinquart M. (2011). A meta-analytic review on the prevention of symptoms of anxiety in children and adolescents. Journal of Anxiety Disorders, 25(8), 1046-1059.

Yoshida, T., Ohtsubo, T., Tsuchida, H., Wada, Y., Kamijima, K., \& Fukui, K. (2004). Development of Sheehan Disability Scale (SDISS) and its reliability and validity. Japanese Journal of Rinsyo-Seishin-Yakuri, 7, 1645-1653.

\section{Tables}

Table 1 Symptom change for the proof of concept study 


\begin{tabular}{|c|c|c|c|c|c|c|c|c|}
\hline & \multicolumn{2}{|c|}{ Pre } & \multicolumn{2}{|c|}{ Week 1} & \multicolumn{2}{|c|}{ Month 1} & $\begin{array}{c}\text { Pre-- } \\
\text { Week } 1\end{array}$ & $\begin{array}{c}\text { Pre- } \\
\text { Month } 1\end{array}$ \\
\hline & Mean & $S D$ & Mean & $S D$ & Mean & $S D$ & & \\
\hline SIAS & 41.00 & 11.89 & 36.33 & 11.09 & 28.67 & 11.88 & -4.67 & -7.66 \\
\hline SPS & 15.17 & 11.44 & 16.17 & 10.23 & 9.83 & 5.60 & 1.00 & -5.34 \\
\hline BDI-II & 5.50 & 2.59 & 3.17 & 2.86 & 1.83 & 1.80 & -2.33 & -3.67 \\
\hline SDS & 9.83 & 5.80 & 5.17 & 2.22 & 4.17 & 2.04 & -4.66 & -5.66 \\
\hline SB & 76.17 & 16.53 & 70.67 & 13.84 & 62.50 & 18.60 & -5.50 & -13.67 \\
\hline
\end{tabular}

Note: SIAS = Social Anxiety Interaction Scale, SPS=Social Phobia Scale, BDI- $\square=$ Beck Depression Inventory, SDS $=$ Sheeha Disability Scale, $\mathrm{SB}=$ Subtle Avoidance Frequency Examination

Table 2 Descriptive statistics for outcome measures

\begin{tabular}{|c|c|c|c|c|}
\hline & & $\begin{array}{c}\text { Pre } \\
\text { Mean }(S D)\end{array}$ & $\begin{array}{c}\text { Week } 1 \\
\text { Mean }(S D)\end{array}$ & $\begin{array}{c}\text { Month } 1 \\
\text { Mean }(S D)\end{array}$ \\
\hline \multirow[t]{2}{*}{ SIAS } & SAFE & $47.12(8.83)$ & $40.15(11.60)^{*}$ & $35.92(12.66)^{* *}$ \\
\hline & HEAL & 49.64 (12.79) & 47.61 (14.88) & $47.52(16.03)$ \\
\hline \multirow[t]{2}{*}{ SPS } & SAFE & 47.85 (14.90) & $41.50(12.18)$ & 20.54 (13.53) \\
\hline & HEAL & $47.55(12.54)$ & $46.52(16.10)$ & $24.10(16.78)$ \\
\hline \multirow[t]{2}{*}{ BDI-II } & SAFE & 18.58 (5.91) & $12.69(7.27)$ & 10.69 (9.23) \\
\hline & HEAL & $19.52(8.96)$ & $14.73(11.64)$ & $14.82(12.89)$ \\
\hline \multirow[t]{2}{*}{ SDS } & SAFE & $8.85(6.69)$ & 7.15 (5.55) & $6.92(5.41)$ \\
\hline & HEAL & $9.36(7.13)$ & $8.12(7.58)$ & 7.76 (7.17) \\
\hline \multirow[t]{2}{*}{ SB } & SAFE & 81.54 (16.33) & 78.73 (15.49) & $69.92(16.30)$ \\
\hline & HEAL & 83.46 (16.76) & 83.76 (19.32) & $77.28(21.24)$ \\
\hline
\end{tabular}

Note: SIAS, Social Interaction Anxiety Scale; BDI, Beck Depression Inventory; SAFE, Subtle Avoidance Frequency Examination; SDS, Sheehan Disability Scale, *=Significant between group differences from pre to Week 1 and pre to Month $1 ;{ }^{* *} p<$ $.01 ;{ }^{*} p<.05$. 
Table 3 Linear Mixed Model of the outcome measures.

\begin{tabular}{|c|c|c|c|c|c|}
\hline Outcomes & Fixed effects & Coefficient & $\mathrm{SE}$ & $95 \% \mathrm{CI}$ & $p$ \\
\hline \multirow[t]{2}{*}{ SIAS } & Group $\times$ post & -4.93 & 2.26 & -9.35 to -0.51 & .029 \\
\hline & Group $\times$ FU & -8.50 & 2.79 & -13.96 to -3.04 & .002 \\
\hline \multirow[t]{2}{*}{ SPS } & Group $\times$ post & -5.32 & 4.41 & -13.95 to 3.32 & .228 \\
\hline & Group $\times$ FU & -4.08 & 4.49 & -12.88 to 4.72 & .363 \\
\hline \multirow[t]{2}{*}{ BDI-II } & Group $\times$ post & -1.10 & 1.43 & -3.90 to 1.71 & .443 \\
\hline & Group $\times$ FU & -3.55 & 1.95 & -7.37 to 0.27 & .068 \\
\hline \multirow[t]{2}{*}{ SDS } & Group $\times$ post & -0.45 & 1.19 & -2.79 to 1.89 & .706 \\
\hline & Group $\times$ FU & 0.01 & 1.22 & -2.38 to 2.39 & .994 \\
\hline \multirow[t]{2}{*}{ SB } & Group $\times$ post & -3.11 & 2.84 & -8.67 to 2.45 & .273 \\
\hline & Group $\times$ FU & -6.20 & 3.29 & -12.65 to 0.25 & .060 \\
\hline
\end{tabular}

Note: SIAS $=$ Social Anxiety Interaction Scale, SPS=Social Phobia Scale, BDI- $\square=$ Beck Depression Inventory, SDS = Sheehan Disability Scale, SB= Subtle Avoidance Frequency Examination, SE= Standard Error, $\mathrm{CI}=$ Confidence Interval. $g=$ Hedge's $g$ between effect sizes. 
Table 4 Effect sizes of outcomes

\begin{tabular}{|c|c|c|c|c|c|}
\hline \multirow[b]{2}{*}{ Outcome } & \multirow[b]{2}{*}{ Group } & \multicolumn{4}{|c|}{ Within ES } \\
\hline & & \multicolumn{2}{|c|}{ Pre to post } & \multicolumn{2}{|c|}{ Pre to FU } \\
\hline & & $g$ & $95 \% \mathrm{CI}$ & $g$ & $95 \% \mathrm{CI}$ \\
\hline \multirow[t]{2}{*}{ SIAS } & SAFE & 0.67 & 0.11 to 1.21 & 1.01 & 0.44 to 1.58 \\
\hline & HEAL & 0.15 & -0.33 to 0.62 & 0.18 & -0.30 to 0.66 \\
\hline \multirow[t]{2}{*}{ SPS } & SAFE & 0.46 & -0.09 to 1.00 & 1.89 & 1.23 to 2.53 \\
\hline & HEAL & 0.07 & -0.41 to 0.55 & 1.19 & 0.66 to 1.70 \\
\hline \multirow[t]{2}{*}{ BDI-II } & SAFE & 0.88 & 0.31 to 1.43 & 1.00 & 0.43 to 1.57 \\
\hline & HEAL & 0.46 & -0.03 to 0.94 & 0.40 & -0.83 to 0.88 \\
\hline \multirow[t]{2}{*}{ SDS } & SAFE & 0.27 & -0.27 to 0.81 & 0.31 & -0.23 to 0.85 \\
\hline & HEAL & 0.17 & -0.31 to 0.64 & 0.25 & -0.23 to 0.73 \\
\hline \multirow[t]{2}{*}{ SB } & SAFE & 0.17 & -0.36 to 0.71 & 0.70 & 0.15 to 1.25 \\
\hline & HEAL & -0.02 & -0.49 to 0.46 & 0.25 & -0.23 to 0.72 \\
\hline
\end{tabular}

Keys: SIAS = Social Anxiety Interaction Scale, SPS=Social Phobia Scale, BDI- $\square=$ Beck Depression Inventory, SDS = Sheehan Disability Scale, SAFE = Subtle Avoidance Frequency Examination, ES= Hedge's g effect sizes, CI= Confidence Interval.

\section{Figures}




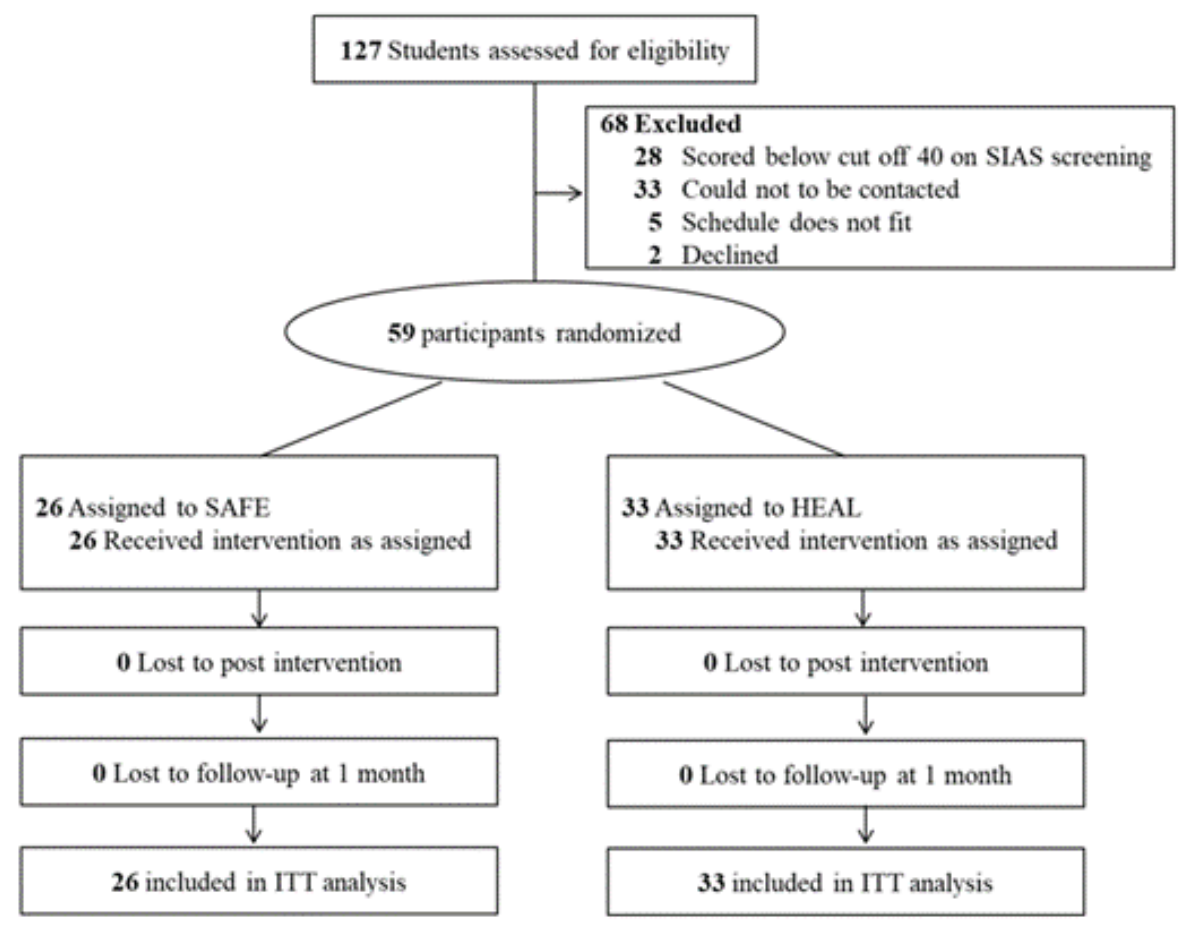

Figure 1

CONSORT diagram of the study.

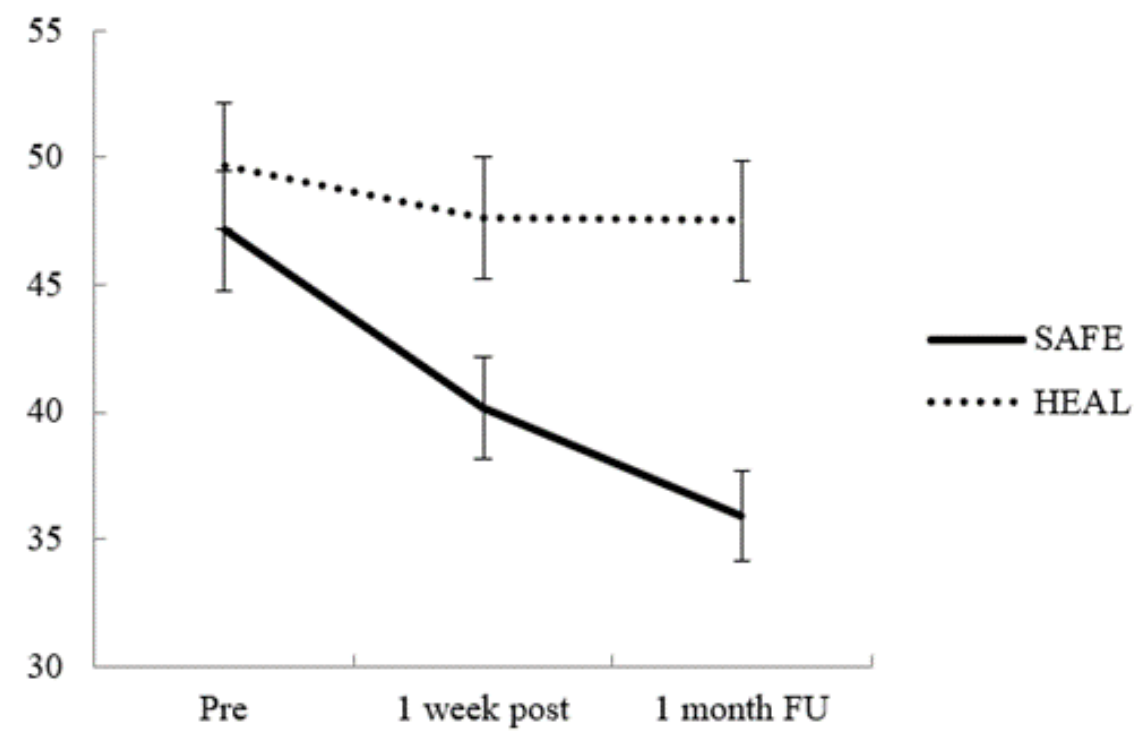

Figure 2

Change in the primary outcome (SIAS scores) over time between groups. 